\title{
Endovascular repair of a life-threatening radiation-induced ruptured false aneurysm of the intrathoracic left subclavian artery: case report
}

This article was published in the following Dove Press journal:

Vascular Health and Risk Management

3I March 2015

Number of times this article has been viewed

\section{Pascale Hizette \\ David Horn \\ Jean Lemaitre \\ Bernard Segers \\ Department of Vascular and Thoracic Surgery, St Pierre University Hospital, Brussels, Belgium}

\begin{abstract}
Massive hemorrhage in tracheostomy patients is generally described as a result of a tracheoinnominate artery fistula. Other etiologies for rupture of a false aneurysm are rare. The classical procedure for subclavian artery aneurysm is open surgery. Endovascular techniques have been accepted by several authors as a possible minimally invasive alternative. We report a life-threatening radiation-induced ruptured false aneurysm of the intrathoracic subclavian artery successfully treated by endovascular stent graft through left brachial access in a tracheostomy patient.
\end{abstract}

Keywords: tracheostomy hemorrhage, radiation-induced aneurysm, subclavian artery, ruptured aneurysm, endovascular repair

\section{Introduction}

Tracheostomy patients undergoing radiotherapy may present acute or late complications such as airway obstruction, hemorrhage, pseudo aneurysm, and infection. Hemorrhage due to surgery or tracheostomy most commonly occurs between the first and third postoperative week and may be caused by injury to blood vessels by radiation. Those hemorrhages are generally controlled with local hemostatic measures. ${ }^{1}$ The worst hemorrhages are the result of a tracheoinnominate artery fistula, which is a rare but life-threatening complication occurring in less than $1.5 \%$ of tracheostomies. ${ }^{1}$ Other etiologies of massive bleeding in tracheostomy patients are rare and isolated cases. $^{2}$ We report the case of a patient with a tracheostomy after total laryngectomy for neoplasia who presented a ruptured radiation-induced false aneurysm of the intrathoracic subclavian artery and massive hemoptysis. He was successfully treated via left brachial endovascular access. Subclavian artery aneurysm is a rare entity. The main etiologies are arteriosclerosis and pseudo aneurysm as a result of trauma, surgery, or radiotherapy. ${ }^{2}$ The classical procedure for subclavian artery aneurysm is open surgery. Several authors have reported endovascular techniques as a possible minimally invasive alternative. ${ }^{1,2}$

\section{Case report}

A 55-year-old Caucasian male was admitted to the emergency room with massive hemoptysis. Four years prior to presentation, the patient had been treated for a left hypopharyngeal squamous carcinoma with surgery (total pharyngosubglossolaryngectomy with cervical lymph node removal, left thyroid lobectomy, and right pectoralis major myocutaneous flap) and radiochemotherapy. Since the end of treatment, he had had regular follow-up. Relevant clinical history included smoking and alcohol abuse.
Correspondence: Bernard Segers Department of Vascular and Thoracic Surgery, St Pierre University Hospital, 322 rue Haute, 1000 Brussels, Belgium Email bernard_segers@stpierre-bru.be submit your manuscript | www.dovepress.com Dovepress 
Daily treatment included L-thyroxine $75 \mu \mathrm{g}$. Clinical examination revealed a cachectic patient with a tracheostomy and multiple surgical scars on the chest and left cervical area. Arterial blood pressure was 90/60 mmHg in both arms and the radial and ulnar pulses were palpated bilaterally. The laboratory findings showed severe anemia (hemoglobin/ dL $8.2 \mathrm{~g}$ ). Computed tomographic (CT) angiography revealed an intrathoracic ruptured false aneurysm $(54 \mathrm{~mm})$ of the left subclavian artery with contrast in both main stem bronchi (Figure 1). While the $\mathrm{CT}$ angiography was being performed, the hemodynamic status of the patient worsened, and he was transferred to the operating room where cardiopulmonary resuscitation was started.

We performed a left surgical brachial artery approach. A 6 French introducer sheath was placed in the left brachial artery. A noncompliant balloon $6 \mathrm{~mm}$ in diameter (Wanda $6 \mathrm{~mm} / 40 \mathrm{~mm}$, Boston Scientific, Fremont, CA, USA) was deployed to stop the bleeding of the ruptured subclavian artery temporarily. The patient's blood pressure improved immediately. The balloon was removed and a $7 \mathrm{~mm}$ diameter, $4 \mathrm{~cm}$ length V12 covered stent (Advanta ${ }^{\mathrm{TM}}$ Atrium Medical Corporation, Hudson, NH, USA) was positioned on the arterial lesion. Angiography during surgery showed successful exclusion of the ruptured aneurysm with no endoleak and preservation of the left vertebral and left internal mammary artery (Figure 2A and B). Operative time was 60 minutes. A drain was left in the brachial access wound for 24 hours. No heparin was administered.

The patient was admitted to the intensive care unit. The predominant clinical manifestations were hypoxemia with metabolic acidosis. The patient underwent several bronchial aspirations. No bronchial infection was noted during his postoperative course. He progressively recovered normal pulmonary function, was discharged from the intensive care unit on day 20, and was discharged home on day 50. CT angiography at one week (Figure 3 ) and one month showed

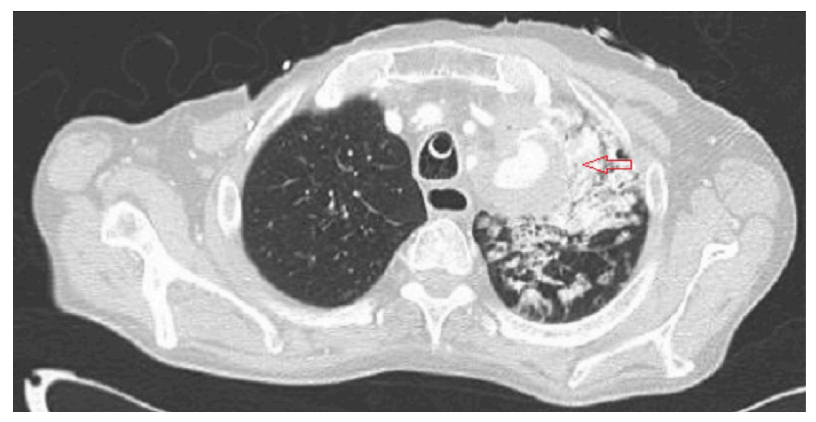

Figure I Preoperative computed tomographic angiography. Arrow indicates a ruptured false aneurysm of the left subclavian artery.
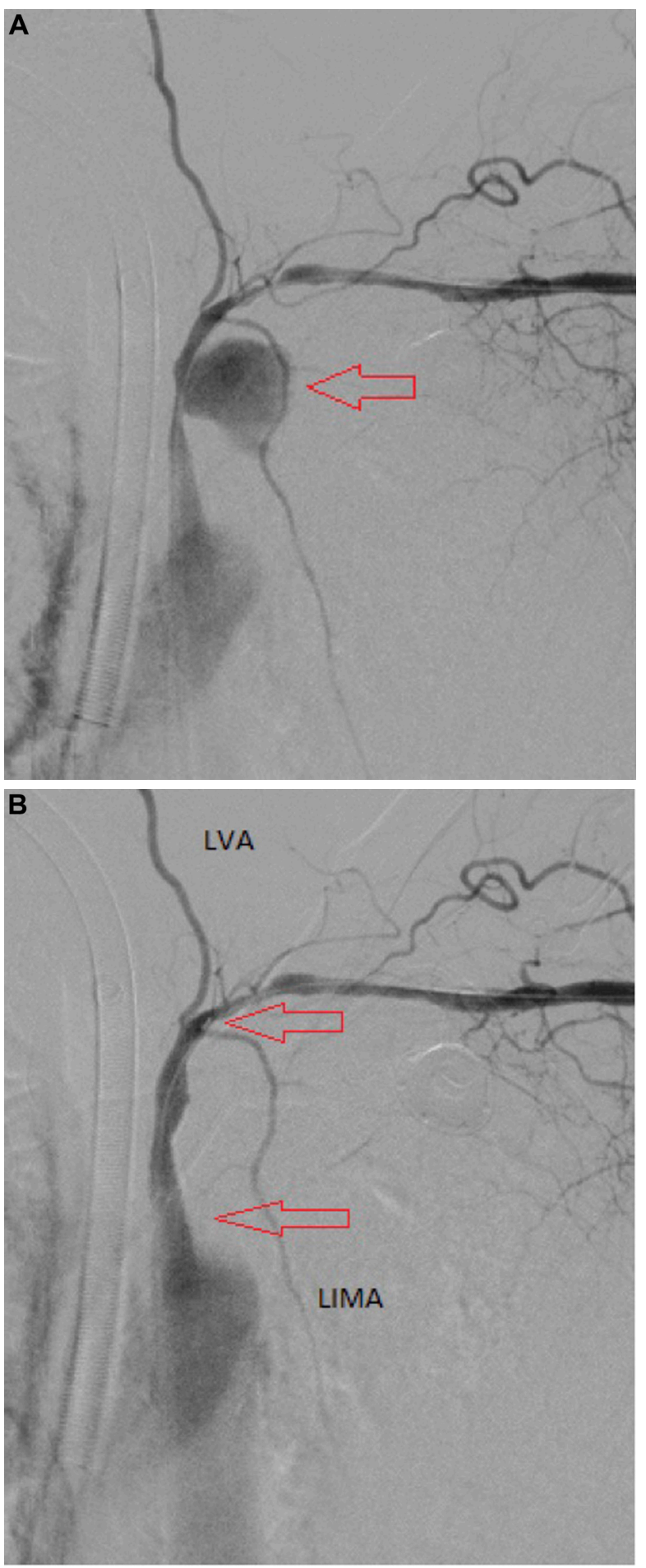

Figure 2 Perioperative angiograms. Arrows indicate (A) aneurysmal bag and (B) covered stent.

Abbreviations: LVA, left vertebral artery; LIMA, left internal mammary artery.

exclusion of the aneurysm, patency of the covered stent, and downsizing of the diameter of the aneurysmal sac. Follow-up is now out to more than 3 years (Figure 4). No complementary surgery has been necessary during follow-up. There has been no recurrence of his cancer. 


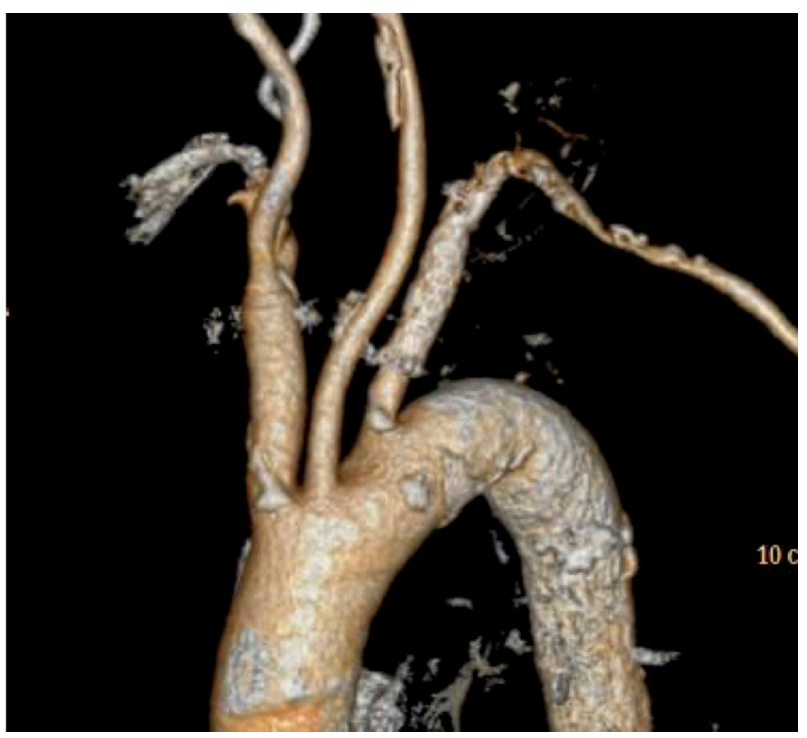

Figure 3 Three-dimensional reconstruction after surgery.

\section{Discussion}

Radiation injury to supra-aortic vessels is a rare and late complication of radiotherapy in the treatment for head and neck or breast cancer. Lesions may be stenotic or aneurysmal. The physiopathology includes occlusion of the vasa vasorum leading to vascular fibrosis, intimal thickening, and endothelial damage, which can accelerate atherosclerosis and pseudo aneurysm formation. ${ }^{3}$ The classical procedure for subclavian artery aneurysm or occlusive lesions is open surgery. The first treatment of subclavian aneurysm was common carotid and innominate artery ligation, performed by Wright Post in 1817 and then by Valentine Mott a year later. ${ }^{4}$ Since then, with the advent of modern surgery, the cervical approach with possible extension to a sternotomy is usually performed. There are not

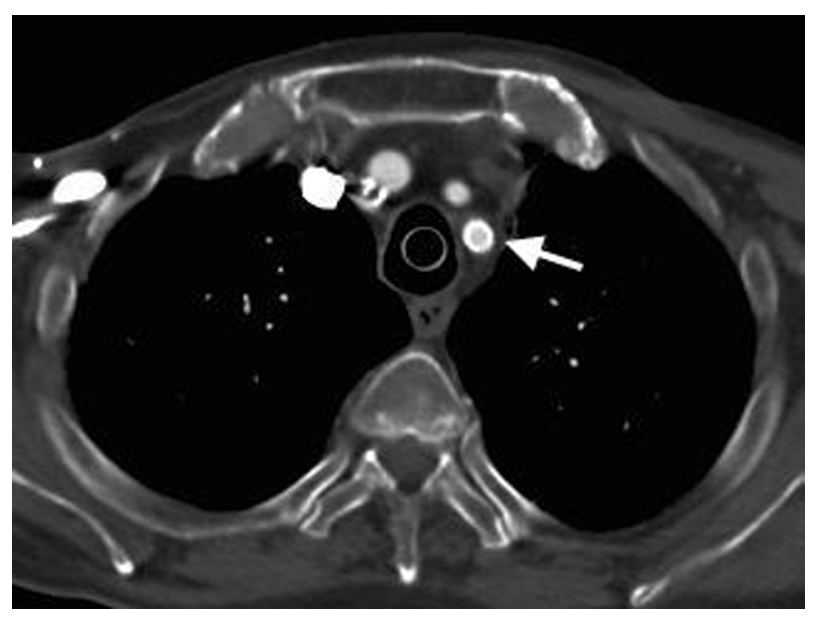

Figure 4 Follow-up computed tomographic angiography at 3 years. Note: Arrow indicates covered stent. many reports on the outcomes of endovascular treatment of ruptured subclavian pseudo aneurysm. Endovascular techniques have been accepted by several authors as a minimally invasive alternative. ${ }^{3,5}$ They can be a better approach because of the lower risk of wound infection and the fragility of the vessels involved. In open surgery, dissection of the irradiated tissue and adequate wound healing is difficult. ${ }^{5}$ Further, the patient described in this report would probably not have tolerated sternotomy. Endograft infection is a rare complication after abdominal or thoracic endovascular repair. But endovascular procedure is now more regularly performed and so the number of endograft infections is increasing, which carries significant associated morbidity and mortality. Surgical excision is the standard of care in this case. Intravenous antibiotics may be of benefit in patients with abdominal endograft infection.

The perioperative morbidity and mortality are lower in elective surgery but increase dramatically in the event of a ruptured aneurysm. ${ }^{4}$ Radiation-induced arterial disease is generally considered to have a poor prognosis and a high risk for infection. ${ }^{3}$ In our case, the open surgery option was a high-risk one because of hypovolemic shock, hypoxemia, and difficult subclavian artery access due to previous surgery and radiotherapy. We opted for an endovascular approach with left brachial artery access. Easy access to the peripheral artery allowed rapid endovascular control of the bleeding artery. The good clinical outcome for the patient in the immediate postoperative period and his 3-year survival indicates that rapid access to the brachial artery and the endovascular approach was the appropriate choice. It allowed rapid occlusion of the bleeding artery prior to positioning a covered stent. In light of this experience, we suggest the endovascular approach in patients with a ruptured aneurysm or false aneurysm of a supra-aortic artery.

\section{Acknowledgment}

The authors thank Marc Derluyn, who encouraged them in the way they chose to treat this patient.

\section{Disclosure}

This case report was presented as a poster at the annual meeting of the International Society for Vascular Surgery, March 9-11, 2012, Miami, FL, USA. The authors have no conflicts of interest to declare in respect of this work.

\section{References}

1. Hackeling T, Triana R, Ma OJ, Shockley W. Emergency care of patients with tracheostomies: a 7-year review. Am J Emerg Med. 1998;16(7): 681-685. 
2. Hung JJ, Hsu HS, Huang CS, Yang KY. Tracheoesophageal fistula and tracheo-subclavian artery fistula after tracheostomy. Eur J Cardiothorac Surg. 2007;32(4):676-678.

3. Ewings EL, Wittgen CM, Paletta CE. Prolonged success with a covered endovascular stent after emergent use in radiation-induced subclavian artery blowout: a case report. Vasc Endovascular Surg. 2008;42(2): 187-191.
4. Garrison FH. Valentine Mott. Bull N Y Acad Med. 1925;1(6):209-214.

5. Hanakita S, Iijima A, Ishikawa O, Kamada K, Saito N. Treatment of a cervical carotid pseudoaneurysm that occurred years after laryngectomy and irradiation of a neck tumor. Neurol Med Chir (Tokyo). 2011;51(8):588-591.

\section{Publish your work in this journal}

Vascular Health and Risk Management is an international, peerreviewed journal of therapeutics and risk management, focusing on concise rapid reporting of clinical studies on the processes involved in the maintenance of vascular health; the monitoring, prevention and treatment of vascular disease and its sequelae; and the involvement of metabolic disorders, particularly diabetes. This journal is indexed on PubMed Central and MedLine. The manuscript management system is completely online and includes a very quick and fair peer-review system, which is all easy to use. Visit http://www.dovepress.com/ testimonials.php to read real quotes from published authors.

Submit your manuscript here: http://www.dovepress.com/vascular-health-and-risk-management-journal 